\title{
La unidad de corta estancia de urgencias y la hospitalización a domicilio como alternativas a la hospitalización convencional
}

\section{The emergency department short stay unit and the hospital at home as alternatives to standard inpatient hospitalisation}

\author{
G. Alonso' ${ }^{1}$, J. M. Escudero ${ }^{2}$
}

\section{RESUMEN}

La unidad de corta estancia de urgencias (UCEU) es una unidad de soporte del servicio de urgencias y una alternativa clara a la hospitalización convencional de pacientes con requerimiento de atención urgente y que están afectados de algunas enfermedades crónicas reagudizadas (estacionales o no) o de patologías de gravedad leve-moderada de corta evolución. La hospitalización a domicilio (HaD) es una alternativa asistencial que permite realizar en el domicilio del enfermo los cuidados y tratamientos propios del hospital, con mayor comodidad e intimidad para éste, y el origen del paciente puede ser tanto la hospitalización convencional, como el servicio de urgencias o la UCEU. En ambas alternativas a la hospitalización convencional, es clave la selección adecuada de los pacientes, mediante criterios estrictos tanto clínicos como sociofamiliares. Su correcto uso asegura un significativo ahorro de estancias en las unidades de hospitalización convencional, una reducción de la estancia media en los servicios médicos y quirúrgicos hospitalarios y una menor presión de urgencias, ya que aumenta la disponibilidad de camas tanto para el ingreso programado como para el ingreso urgente en hospitalización convencional y mejora, por tanto, el drenaje del servicio de urgencias.

Palabras clave. Unidad de corta estancia. Urgencias. Gestión hospitalaria. Hospitalización a domicilio. Alternativa a la hospitalización.

\begin{abstract}
The emergency short stay unit (ESSU) is a support unit for the accident and emergency services and a clear alternative to the conventional hospitalisation of patients needing urgent care, who are affected by the re-aggravation of certain chronic diseases (whether seasonal o not) or by moderate short-evolution pathologies. Hospitalisation at home is one alternative making it possible to undertake the care and treatment characteristic of a hospital in the home of the patient, with greater comfort and intimacy. The origin of the patient might be the conventional hospital, the emergency service or ESSU. A suitable selection of patients is essential in both alternatives to conventional hospitalisation, using strict criteria that are both clinical and socio-family in nature. Their correct use assures a significant saving in stays in conventional hospital units, a reduction of the average stay in hospital medical and surgical services and less pressure on the accident and emergency service, since they increase the availability of beds both for programmed admission and for urgent admission in conventional hospitalisation and thus improve patient outflow in the accident and emergency service.
\end{abstract}

Key words. Short stay unit. Emergency room. Hospital management. Hospital at home. Alternative hospitalisation.
1. Servicio de Urgencias. Hospital Universitari de Bellvitge. L`Hospitalet de Llobregat. Barcelona.

2. Unidad de Hospitalización a Domicilio. Pamplona.

\section{Correspondencia}

Gilberto Alonso Fernández

Servicio de Urgencias

Hospital Universitari de Bellvitge

Feixa Llarga, $\mathbf{s} / \mathbf{n}$

08901 L’Hospitalet de Llobregat. Barcelona.

España.

E-mail: galonso@bellvitgehospital.cat 


\section{UNIDAD DE CORTA ESTANCIA DE URGENCIAS}

\section{Introducción}

El incremento progresivo de la demanda de atención urgente hospitalaria es una realidad constatada en la mayoría de los servicios de urgencias hospitalarios (SUH) hasta el día de hoy. Son la puerta de entrada más importante del hospital (presión de urgencias mayor al $50 \%)^{1}$. Dejando de lado el análisis de las posibles causas externas e internas de dicho aumento de la demanda y que han sido objeto de muchos trabajos ${ }^{2}$, el día a día nos muestra la situación de conflicto que genera la competencia por las camas hospitalarias entre los ingresos procedentes del SUH y los ingresos procedentes de la programación. O lo que es lo mismo, el dilema que se le plantea a los directivos entre acumular pacientes pendientes de ingreso sin cama (con el consiguiente empeoramiento de las condiciones de trabajo en el SUH) o anular pacientes programados (contribuyendo a aumentar las listas de espera fundamentalmente quirúrgicas) ${ }^{3}$.

Entre las soluciones planteadas, las alternativas a la hospitalización convencional (los hospitales de día de diferentes especialidades, las unidades de observación de urgencias, las unidades de diagnóstico rápido, la hospitalización a domicilio o las unidades de corta estancia de urgencias ${ }^{4-5}$, entre otras) han demostrado ser costo-efectivas y facilita el drenaje de los SUH, disminuyen el número de ingresos inadecuados en hospitalización convencional, aumentan la capacidad de ingreso programado o, lo que es lo mismo, disminuyen la presión de urgencias.

\section{Objetivos}

Las principales funciones de la unidad de corta estancia de urgencias (UCEU) consisten en:

1. Dar soporte al SUH, mejorando su drenaje.

2. Evitar ingresos inadecuados en hospitalización convencional, mediante la optimización de las estancias hospitalarias de patología crónica agudizada.
Los objetivos de la UCEU son:

1. Contribuir a paliar el problema de saturación de los $\mathrm{SUH}$, facilitando su drenaje y evitando que la estancia media de los pacientes ya diagnosticados y en tratamiento supere las 24 horas. Esto es, como tercer escalón de la asistencia dentro del SUH después de la primera asistencia, la unidad de observación de urgencias (UOU) y cuando no proceda el ingreso hospitalario convencional. Este apoyo permite disminuir el tiempo de estancia en el SUH, aumenta el índice de rotación de los puntos de asistencia (tanto de primera asistencia como de la UOU) y disminuir el tiempo de espera para ser atendido.

2. Ofrecer una respuesta más adecuada a los diferentes tipos de demanda de atención urgente. Compartimenta la asistencia en el SUH y evita que un paciente (y su familiar o acompañante) que precise de una estancia más prolongada sufra las incomodidades de las zonas de primera asistencia.

3. Establecer alternativas al ingreso convencional en patologías médicas, sobre todo en aquellos pacientes con patología médica agudizada que, si bien necesitan más de 24 horas de tratamiento y observación, pueden beneficiarse de estancias hospitalarias de menos de 72 horas $^{6}$.

4. Disminuir el impacto de los ingresos urgentes sobre la actividad programada. Ésta es el resultado de las anteriores; una menor competencia por la cama de hospitalización convencional (menor presión de urgencias) facilita la entrada de la actividad programada $y$, a su vez, junto con otras medidas directivas que no son objeto de este artículo, produce el efecto paradójico de aumentar la disponibilidad de camas para el ingreso urgente.

Para que la UCEU pueda cumplir los objetivos de forma eficaz es fundamental una correcta selección de los pacientes tributarios de ingreso en la misma y una correcta 
información a pacientes y familiares de las particularidades y objetivos de la UCEU ${ }^{7}$.

\section{Criterios de ingreso}

Los pacientes que ingresen en la UCEU deben cumplir los siguientes criterios ${ }^{8}$ :

1. Pacientes diagnosticados en el SUH y cuya estancia previsible sea menor a 72 horas.

2. Pacientes con necesidades mínimas de pruebas complementarias, la mayoría de las cuales deberían haber sido realizadas en el SUH previamente al ingreso en la UCEU.

3. Pacientes con procesos agudos ya diagnosticados que su período de observación y evolución haya superado las 24 horas y, por tanto, no deban permanecer más tiempo en la UOU, facilitando el drenaje de la misma y del resto del SUH.

4. Pacientes que puedan ser alta a domicilio, o beneficiarse también de control domiciliario de atención primaria, control por equipo de hospitalización a domicilio y traslado a un centro de subagudos de soporte.

Tan importantes, o más, que los criterios de ingreso son los criterios de no ingreso:

1. Pacientes que tras su estancia en el SUH no tienen una orientación diagnóstica clara.

2. Pacientes con probable problemática social y/o familiar al alta.

3. Pacientes que requieran aislamiento.

4. Pacientes que puedan requerir exploraciones complementarias complejas.

5. Pacientes que puedan presentar inestabilidad hemodinámica.

6. Pacientes programados o pacientes que deberían ingresar en una unidad de hospitalización convencional de otras especialidades.

Si bien los motivos fundamentales de ingreso en la mayoría de las UCEU son la insuficiencia cardíaca descompensada ${ }^{5}$ y la EPOC descompensada ${ }^{9}$, la UCEU puede tratar cualquier tipo de patología urgente siempre y cuando cumpla los criterios previos. Por esta razón, es más coherente definir los criterios en términos de estancia y necesidades que en forma de un largo listado de patologías, dado que la UCEU puede abarcar la observación y tratamiento de un gran número de ellas ${ }^{4-5}$.

\section{Control de calidad}

El control de calidad de la UCEU se centra fundamentalmente en dos aspectos ${ }^{9}$ : correcta selección de pacientes desde el SUH y correcto manejo de los mismos desde la UCEU.

Son pocos los indicadores necesarios para evaluar la efectividad de una UCEU, siendo los fundamentales:

1. Estancia media: la ideal es $\leq 3$ días.

2. Índice de ocupación: alrededor del $90 \%$.

3. Índice de destino al alta externo: el ideal es $\geq 90 \%$.

4. Índice de retorno a unidad de hospitalización convencional: el ideal es $\leq 10 \%$.

5. Tasa de mortalidad no esperada: la ideal es $\leq 1 \%$.

6. Índice de reconsultas al SUH dentro de las 72 horas del alta de la UCEU: el ideal es $\leq 5 \%$.

\section{Organización dentro del SUH}

Jerárquicamente la UCEU, al igual que el resto de unidades del SUH, debe ser responsabilidad del jefe de Servicio de Urgencias. Dependiendo del tamaño del SUH y de la UCEU, es conveniente que exista un responsable directo de la unidad (jefe de unidad o jefe clínico) y sus facultativos deben estar adscritos al SUH.

La distribución de la jornada laboral de los facultativos de urgencias en la UCEU no debe variar de la del resto del SUH. Es el ideal el modelo de turnos, que garantiza la continuidad asistencial y el mantenimiento de la organización del servicio en relación 
al tiempo de trabajo y al descanso laboral ${ }^{9}$. Es conveniente la superposición de equipos de mañana (8-15h) y tarde (13-20h), que asegura una mayor actividad (número de altas) durante las horas de máxima actividad del SUH. La relación camas/médico debe ser alrededor de ocho, habida cuenta del alto índice de rotación de la unidad, y resulta ideal el trabajo en equipo con una relación similar de enfermería.

El perfil del médico de la UCEU ha de ser exactamente el mismo que el del SUH, un experto en medicina de urgencias ${ }^{10}$. Es aconsejable que forme parte de una rotación más dentro del SUH, aspecto que, por un lado, ayuda a consolidar el buen uso de la unidad y, por otro, descarga al profesional del SUH de estar siempre en primera línea.

Respecto a la actividad de la misma, si bien es conveniente disponer de una UCEU la totalidad del año, el planteamiento de la misma en términos de períodos de apertura, de número de camas y de dotación de personal, dependerá de las necesidades propias de cada centro. Es fácil calcular el soporte que puede dar tanto al SUH en primera instancia, como al resto del hospital. La disminución de la presión de urgencias, la disminución del tiempo de estancia media en urgencias y la disminución de la estancia media hospitalaria serán los indicadores principales.

\section{Consideraciones finales}

En resumen, la UCEU es una unidad eficaz y segura en la atención de pacientes con patología médica tributaria exclusivamente de tratamiento y evolución en un máximo de 72 horas. No es una unidad de diagnóstico o de preingreso en otros servicios de hospitalización convencional, su función es de soporte del SUH facilitando el drenaje del mismo desde una doble vertiente: la inmediata como tercer escalón dentro del SUH y diariamente como facilitador de mayor disponibilidad de camas de hospitalización convencional. La instauración de UCEU ha significado una alternativa a la hospitalización convencional, que ahorra estancias en dicha hospitalización con- vencional, disminuye la estancia media de los servicios médicos hospitalarios ${ }^{11} \mathrm{y}$, por ende, disminuye la presión de urgencias y la competencia entre la cama urgente y la programada. La UCEU es una unidad totalmente compatible con otras alternativas a la hospitalización convencional: hospitalización a domicilio, hospitalización en centros de subagudos o alta a domicilio con control domiciliario de atención primaria. Se potencia con todas ellas y evita el retorno o reconsulta en el SUH ${ }^{12}$.

\section{HOSPITALIZACIÓN A DOMICILIO}

\section{Introducción}

La aparición de nuevos fármacos, los avances de la cirugía mínimamente invasiva, el desarrollo de las tecnologías de la información, la miniaturización de aparatos y las nuevas fórmulas de gestión están modificando las actuales pautas de asistencia y van a generar en el futuro grandes cambios en el sector de la salud y muy especialmente en la organización de los hospitales ${ }^{13,14}$. En este contexto, se plantea la necesidad de transformar y reorganizar los sistemas y servicios de salud desarrollando diferentes programas y alternativas institucionales, ambulatorias y domiciliarias, encaminadas a mejorar la situación sanitaria ${ }^{15}$. Una de estas alternativas es la hospitalización a domicilio (HaD) que podemos definir como una alternativa asistencial capaz de proporcionar en el domicilio, a determinados pacientes, niveles de diagnóstico, cuidados y tratamiento similares a los dispensados en el hospital. En 1947 se creó en el hospital Montefiore de Nueva York la primera unidad de hospitalización a domicilio (UHD). En Europa se inició en 1951, concretamente en el hospital Tenon de París, y se extendió progresivamente por el resto del continente. Esta modalidad asistencial llegó a España en 1981 cuando comenzó a funcionar la unidad del actual hospital Gregorio Marañón. En la década de los noventa las UHD tuvieron un gran auge en nuestro país y llegó paulatinamente a la mayoría de las comunidades autónomas ${ }^{16,17}$. 
El modelo asistencial de la HaD varía según los países. Así, mientras en el Reino Unido la asistencia se relaciona más con la provisión de servicios sociosanitarios a través del médico de Atención Primaria y de personal de enfermería ${ }^{18,19}$, en otros países europeos y en EEUU se ha desarrollado para suministrar una asistencia más compleja similar a la hospitalaria ${ }^{20}$.

De la definición de la $\mathrm{HaD}$ se derivan una serie de características ${ }^{13,21}$ :

1. Transitoriedad: el seguimiento se limita al tiempo que transcurre hasta la resolución del proceso agudo que motivó su ingreso.

2. Complejidad: por el personal especializado que trabaja en estas unidades y por la utilización de técnicas diagnósticas y terapéuticas propias del hospital ${ }^{14}$.
3. Intensidad: habitualmente se realiza una visita diaria a los pacientes pero en ocasiones requieren varias actuaciones diarias.

4. Trabajo en equipo: el personal sanitario de la unidad (médicos-enfermería) trabaja de forma conjunta y coordinada. Además, es necesaria una coordinación con los diversos niveles asistenciales implicados en la atención de los enfermos.

La HaD no es un servicio adecuado para realizar un seguimiento de pacientes cróni$\cos$, que precisan de otro estamento asistencial como es la atención primaria. Tampoco la $\mathrm{HaD}$ es la solución de la saturación que puedan sufrir los hospitales, aunque puede ser una ayuda para minimizar los inconvenientes derivados de estas situaciones ${ }^{22}$.

En la tabla 1 se detallan las diferencias entre la atención proporcionada desde la $\mathrm{HaD}$ y desde la atención primaria ${ }^{23}$.

Tabla 1. Atención domiciliaria. Características diferenciadoras.

\begin{tabular}{lll}
\hline & \multicolumn{1}{c}{ HaD } & Atención Primaria \\
\hline Misión & Hospital & Centro Salud \\
\hline Cuidados & Salud & Salud \\
Tipo & Alta & Baja \\
Intensidad & Alta & Baja \\
Complejidad & Transitoria & Larga \\
Duración de la atención & Sí & No \\
\hline Tecnología & & \\
Características de los pacientes & Cualquiera & $>65$ \\
Edad & Cualquiera & Crónicos \\
Patologías & Sí/no & Sí \\
Dependencia & Agudo & Estable \\
Estado evolutivo enfermedad & Pública & Pública \\
\hline Financiación & Pública & Pública \\
\hline Provisión & Hospital & Atención Primaria \\
\hline Origen de los recursos & &
\end{tabular}

\section{Dependencia jerárquica}

Los modelos de $\mathrm{HaD}$ implantados en nuestro país dependen jerárquicamente de diversos estamentos, como gerencia, direc- ción médica, servicios de medicina interna, SUH, dirección de atención especializada, unidades independientes, etc. Cualquiera de estas fórmulas puede ser correcta. Las dependientes de servicios concretos tie- 
nen la ventaja de disponer de un flujo constante de pacientes desde dicho servicio, pero puede limitarse el ingreso desde otras unidades. Para evitar este problema, la experiencia muestra que la mejor fórmula sea probablemente la dependencia de un estamento superior a un servicio concreto ${ }^{24}$.

\section{Organización de las unidades de hospitalización a domicilio}

Las UHD están dirigidas por un responsable, que dependiendo de la organización de cada hospital, ostenta la categoría de jefe de servicio, jefe de sección o coordinador. Cada unidad debe de disponer al menos de un médico que realice las labores asistenciales en el domicilio. Generalmente son especialistas en medicina interna o medicina familiar y comunitaria, aunque también pueden incorporar especialistas como neumólogos, geriatras, cirujanos generales, rehabilitadores, etc. El personal de enfermería ha de estar formado en diversas especialidades hospitalarias, tanto médicas como quirúrgicas, para dar una asistencia de calidad. Cada equipo de trabajo está formado por un médico y una enfermera que tiene la responsabilidad de entre 8 y 10 pacientes ingresados. En general la relación cuantitativa del personal sanitario en las UHD es de 2 ó 3 enfermeras por cada médico, en función de la carga de pacientes que traten las enfermeras en solitario (curas, tratamientos endovenosos, realización de pruebas diagnósticas, etc.). Las visitas en el domicilio se realizan todos los días del año, habitualmente en horario de mañana, quedando posteriormente parte del personal de guardia para garantizar la continuidad asistencial del enfermo.

\section{Modalidades de hospitalización a domicilio}

El concepto de $\mathrm{HaD}$ engloba diferentes niveles o esquemas asistenciales que permiten atender a pacientes en diferentes fases evolutivas de la enfermedad, por lo que la duración de los cuidados y el consumo de recursos es también distinto. Entre los esquemas asistenciales más habituales encontramos $^{16,23}$.

1. HaD como complemento de la hospitalización convencional en determinados procesos patológicos. El paciente ingresa en régimen de hospitalización convencional durante unos días y una vez estabilizado pasa a ser tratado en HaD hasta ser dado de alta. Esta modalidad se denomina también de alta precoz porque acorta la estancia del enfermo en el hospital.

2. HaD como sustitución de la hospitalización convencional: tras la realización de las pruebas complementarias necesarias, los pacientes son ingresados en la $\mathrm{HaD}$ desde el SUH o desde una UCEU. Mediante esta modalidad, el enfermo ni siquiera llega a ocupar una cama en una planta del hospital.

3. Función de soporte: en este esquema se efectúan en el domicilio, tanto valoraciones clínicas y/o analíticas de rango hospitalario para la gestión de casos complejos, a petición de los equipos de atención primaria. Suelen ser ingresos muy breves.

4. Otra modalidad es la denominada de "alta tecnología", que implica la aplicación de procedimientos diagnósticos y terapéuticos complejos y es equivalente a un hospital de día.

\section{Objetivos y ventajas}

Los objetivos generales de la $\mathrm{HaD}$ incluyen dar cobertura a una necesidad asistencial no atendida por otros niveles asistenciales, asegurar la continuidad asistencial integral una vez el paciente es dado de alta del hospital, actuar de puente o conexión entre el hospital y atención primaria así como optimizar la gestión de los recursos existentes. Las ventajas de la $\mathrm{HaD}$ respecto de la hospitalización convencional pueden agruparse en tres grandes bloques, como se observa en la tabla $2^{16,25}$. 
Tabla 2. Ventajas de la hospitalización a domicilio

\section{VENTAJAS HOSPITALIZACIÓN A DOMICILIO}

\section{Ventajas Sociofamiliares}

- Permite mayor intimidad y comodidad del paciente

- Mejora la relación personal sanitario-paciente

- Facilita la comunicación, a la vez que humaniza dicha relación

- Liberaliza los estrictos horarios hospitalarios para las actividades propias: alimentación, descanso, higiene...

- Integra a la familia en el proceso de curación del paciente

- Evita desplazamientos de la familia al hospital

- Promueve las actividades de educación del personal sanitario en el entorno familiar.

\section{Ventajas técnico-sanitarias}

- Disminuyen las infecciones nosocomiales

- Evitan el hospitalismo

- Disminuyen los episodios de confusión mental y/o depresión en ancianos

Ventajas en gestión
- Sirve de conexión entre el hospital y la atención primaria
- Favorece la utilización de las camas hospitalarias por las personas apropiadas
- Disminuye los costes marginales por proceso

\section{Criterios de ingreso de pacientes}

Un aspecto importante del trabajo diario de las UHD son las valoraciones en la planta de hospitalización y en los SUH de los pacientes susceptibles de ingreso. Esta valoración se realiza tanto desde el punto de vista clínico como sociofamiliar. El ingreso en $\mathrm{HaD}$ conlleva unos criterios que los enfermos han de cumplir y que se reflejan en la tabla $3^{20,17}$.

Tabla 3. Criterios de inclusión en hospitalización a domicilio.

\section{CRITERIOS DE INGRESO}

- Que el proceso que sufre el paciente pueda de ser tratado en el domicilio.

- Aceptación voluntaria del paciente y la familia del ingreso en la $\mathrm{HaD}$.

- Se precisa de un cuidador principal, familiar o no, que colabore con el personal sanitario en los cuidados del enfermo:

- Realizar los cuidados básicos del paciente

- Control y administración de medicación oral

- Realización de tareas sencillas (glucemias capilares, temperatura, etc.)

- Ayuda en el tratamiento (retirada de sueros, lavado de vías, administración de aerosolterapia, etc.)

- La vivienda ha de cumplir unos requisitos mínimos de habitabilidad

- Disponer de línea telefónica que permita contactar en caso necesario

- El paciente debe residir habitualmente o temporalmente dentro del área de cobertura de la UHD

- Que la situación clínica del paciente esté suficientemente compensada para que haga poco probable su empeoramiento

- Ausencia de hábitos tóxicos activos

HaD: hospitalización a domicilio

UHD: unidad de hospitalización a domicilio 


\section{Cartera de servicios}

Entendemos como tal el catálogo de actividades, servicios y prestaciones de un dispositivo asistencial, encuadrados dentro de su entorno social y demográfico. El impulso de las UHD en las distintas co- munidades autónomas no ha sido uniforme debido a que cada unidad ha crecido en un entorno diferente, lo que hace que la cartera de servicios sea también distinta. En la tabla 4 observamos, de forma general, los procedimientos diagnósticos y terapéuticos que se pueden realizar en $\mathrm{HaD}^{16}$.

Tabla 4. Procedimientos realizables en el domicilio.

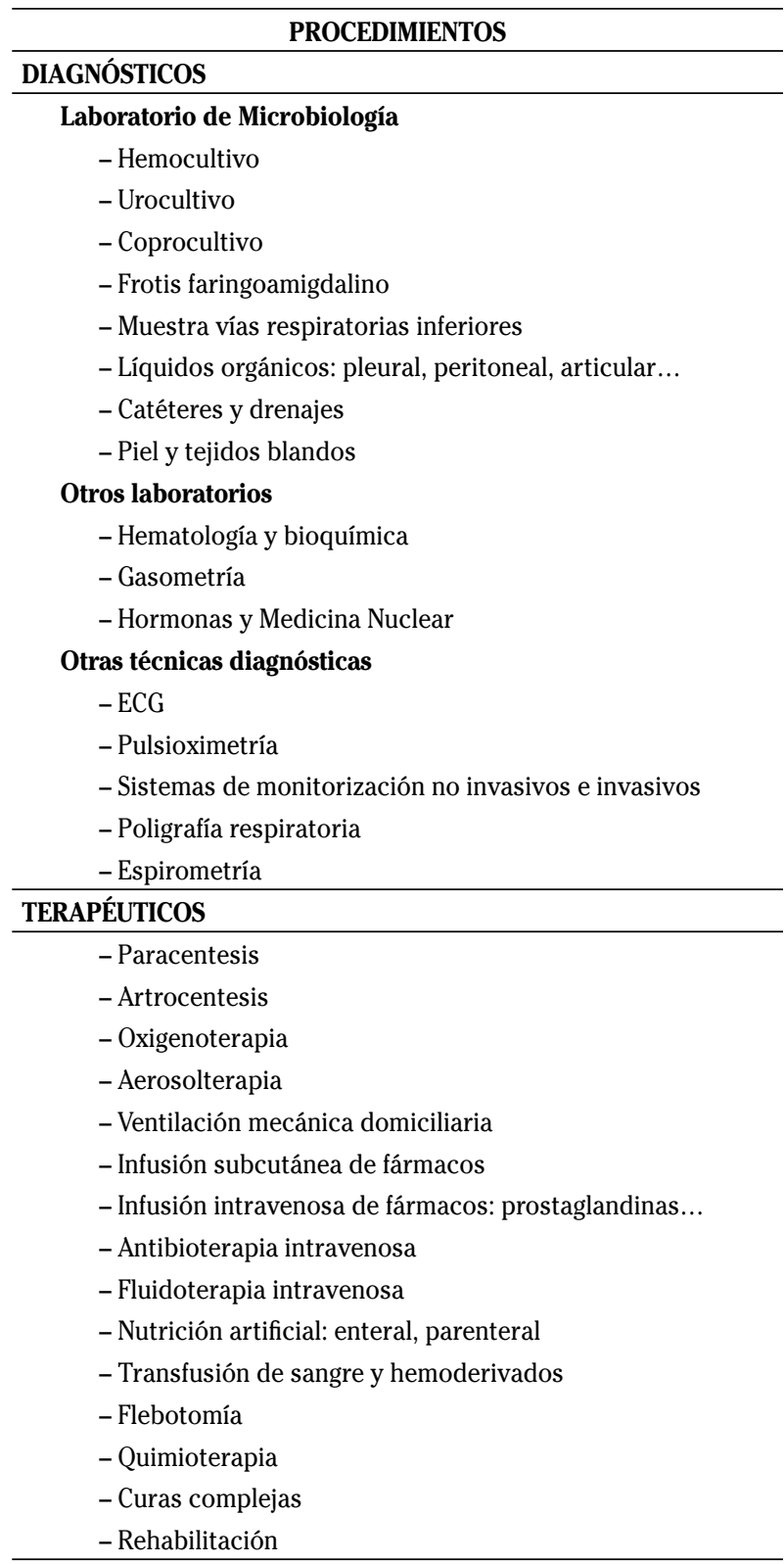




\section{Procesos atendidos en hospitalización a domicilio}

No existe una uniformidad respecto a las enfermedades que se tratan en las distintas UHD, lo cual no es un signo de debilidad, ya que es fruto de la diversidad de orígenes de las unidades, que hace que los procesos sean también distintos. De todos modos, de forma general los procesos tratados en $\mathrm{HaD}$ podemos agruparlos en los siguientes apartados ${ }^{20,25-27}$.

1. Enfermedades infecciosas: neumonías, infecciones urinarias, heridas infectadas, bronquiectasias infectadas, úlceras colonizadas por gérmenes resistentes, celulitis, abscesos de diversos orígenes, etc.

2. Enfermedades crónicas descompensadas: enfermedad pulmonar obstructuva crónica, insuficiencia cardiaca, insuficiencia renal, diabetes mellitus, hepatopatías, etc.

3. Pacientes oncológicos:

- En algunas unidades se realiza tratamiento de neoplasias mediante quimioterapia o seguimiento de pacientes paliativos.

- Enfermedades intercurrentes en enfermos oncológicos: bacteriemias febriles, neutropenias febriles, trombosis venosas, etc.

- Los mismos procesos que la población general, tanto descompensaciones de enfermedades crónicas como agudas.

4. Pacientes de servicios quirúrgicos:

- Tratamiento y seguimiento de postoperatorios: artroplastias de cadera y rodilla, intervenciones abdominales, urológicas, etc.

- Procesos infecciosos ingresados en servicios quirúrgicos, como diverticulitis, colangitis, colecistitis, abscesos, osteomielitis, etc., que requieran antibioterapia endovenosa.

- Curas complejas de heridas quirúrgicas, úlceras de decúbito, úlceras vasculares, etc.
5. Atención de pacientes en situación terminal

6. Atención de enfermos trasplantados: médula ósea, hígado, etc.

7. Otras patologías variables según las unidades.

\section{Consideraciones finales}

La HaD es una alternativa asistencial plenamente establecida en nuestro país, válida para muchos pacientes con diversos procesos patológicos. Debido a los cambios que se están produciendo en los sistemas gerenciales de los hospitales, más centrados en la atención ambulatoria de los pacientes, la $\mathrm{HaD}$ tiene un futuro prometedor al ser un modelo asistencial que oferta al enfermo las mismas posibilidades de diagnóstico y tratamiento que las recibidas en una planta de hospitalización ${ }^{15,24}$. Con respecto a la relación con los $\mathrm{SUH}$, destacar que son una fuente importante de pacientes susceptibles de ingreso en $\mathrm{HaD}$, tanto desde los propios SUH como de las UCEU ${ }^{24}$.

\section{BIBLIOGRAFÍA}

1. GRAFF L. Overcrowding in the Emergency Department: an International symptom of health care system failure. Am J Emerg Med 1999; 17: 208-209.

2. Miró O, SÁnchez M, Coll-Vinent B, Millà J. Estimación del efecto relativo que ejercen los determinantes externos e internos sobre la eficacia de un servicio de urgencias de medicina. Med Clin (Barc) 2000; 115: 294-296.

3. Escarrabill J, Corbella X, Salazar A, Sánchez Jl. Los colapsos en los servicios de urgencias hospitalarios durante el invierno. Aten Primaria 2001; 27: 137-140.

4. Juan A, Salazar A, Álvarez A, Pérez JR, García L, Corbella X. Effectivenes and safety of an Emergency Department short-stay unit as an alternative to standard inpatient hospitalization. Emerg Med J 2006; 23: 833-837.

5. GonzÁlez-Armengol JJ, Fernández Alonso C, Martín-SÁnchez FJ, González-del Castillo J, LóPEZ FARré A, Elvira C et al. Actividad de una unidad de corta estancia de urgencias de un hospital terciario: cuatro años de experiencia. Emergencias 2009; 21: 87-94. 
6. Corbella X, Salazar A, Maiques JM, Juan A. Unidad de corta estancia de urgencias, una alternativa a la hospitalización convencional. Med Clin (Barc) 2002; 118: 515-516.

7. Gómez C, Gulllamont J, Salazar A, Novelli A, Corbella X. Evaluación de la satisfacción de los pacientes ingresados en una unidad de corta estancia de urgencias. Emergencias 2005; 17:12-16.

8. Salazar A, Juan A, Ballbé R, Corbella X. Emergency Short Stay Unit as an effective alternative to in-hospital admission for acute COPD exacerbation. Am J Emerg Med 2007; 25: 486-487.

9. Salazar A, Juan A, Corbella X. Unidades asistenciales de apoyo a urgencias: la unidad de corta estancia de urgencias. Monografías Emergencias 2008; 1: 9-11.

10. Salazar A. ¿Urgenciólogos rentables? Emergencias 2009; 21: 83-84.

11. Kong GK, Belman MJ, Weingarten S. Reducing length of stay for patients hospitalized with exacerbation of COP by using a practice guideline. Chest 1997; 111: 89-94.

12. Hernández C, Casas A, Escarrabill J, Alonso J, Puig-Junoy J, FARrero E et al. CHRONIC project. Home hospitalization of exacerbated chronic obstructive pulmonary disease patiens. Eur Respir J 2003; 21: 58-67.

13. VAQUÉ J. El aumento de la longevidad y su relación con la morbilidad. Med Prev 1998; 4 : 19-26.

14. WiLson CB. The impact of medical technologies on the future of hospitals. BMJ 1999; 319: 1287.

15. Minardi Mitre Cotta R, Morales Suárez-Varela M, Cotta Filho JS, Llopis González A, Días Ricós JA, RAmón ReAL E. La hospitalización domiciliaria ante los cambios demográficos y nuevos retos. Rev Panam Salud Pública/Pan Am J Public Health 2002; 11: 253-261.

16. González Ramallo VJ, Valdivieso Martínez B, Ruiz García V. Hospitalización a domicilio. Med Clin (Barc) 2002; 118: 659-664.
17. Valdivieso Martínez B, Ruiz García V. Hospitalización a domicilio y atención domiciliaria. Hacia la excelencia de los servicios. FMC 2001; 8: 537-542.

18. Mowat IG, Morgan RTT. Peterborough hospital at home scheme. BMJ 1982; 284: 641-643.

19. SHEPPERD S, ILIFFe S. Hospital at home. BMJ 1996; 312: 923-924

20. SHEPPERD S, ILIFFE S. The effectiveness of hospital at home compared with in-patient hospital care: a systematic review. J Publ Hlth Med 1998; 20: 344-350.

21. Minardi Mitre Cotta R, Morales Suárez-Varela M, Llopis González A, CotTa Filho JS, Ramón ReAl E, Días Ricós JA. La hospitalización domiciliaria: antecedentes, situación actual y perspectivas. Rev Panam Salud Pública/Pan Am J Public Health 2001; 10: 45-55.

22. Contel JC. La hospitalización a domicilio no es una alternativa plenamente recomendable. Aten Primaria 1999; 24: 162-168.

23. Valdivieso Martínez B, Ruiz García V. Hospitalización a domicilio y atención domiciliaria. Hacia la excelencia de los servicios. FMC 2001; 8: 537-542.

24. Porta Castejón R, Germán Tomás A, Tomás VeCINA S. Unidades de hospitalización a domicilio como alternativa a la hospitalización convencional: organización y su repercusión en los servicios de urgencias. Monografías emergencias 2008; 1: 12-19.

25. Torres salinas M, Capdevila Morel JA, Armario García P, Montull Morer S y grupo de trabajo de los servicios de medicina interna de los hospitales de Cataluña. Alternativas a la hospitalización convencional en medicina interna. Med Clin (Barc) 2005; 124: 620-626.

26. MARRAdes RM. Hospitalización domiciliaria, ¿una nueva modalidad asistencial? Arch Bronconeumol 2001; 37: 157-159.

27. SHEPPERd S, IlifFe S. Hospital domiciliario versus atención hospitalaria estándar. The Cochrane Library, 2008 issue 1. 